Portland State University

PDXScholar

\title{
Chart Review Observing Depression and Anxiety in a Cohort of Nontuberculous Mycobacteria Patients
}

\author{
Megan N. Feely \\ Portland State University
}

Follow this and additional works at: https://pdxscholar.library.pdx.edu/honorstheses

\section{Let us know how access to this document benefits you.}

\section{Recommended Citation}

Feely, Megan N., "Chart Review Observing Depression and Anxiety in a Cohort of Nontuberculous Mycobacteria Patients" (2019). University Honors Theses. Paper 711.

https://doi.org/10.15760/honors.728

This Thesis is brought to you for free and open access. It has been accepted for inclusion in University Honors Theses by an authorized administrator of PDXScholar. Please contact us if we can make this document more accessible: pdxscholar@pdx.edu. 
Chart Review Observing Depression And Anxiety In A Cohort Of Nontuberculous Mycobacteria Patients

by

Megan Feely

An Undergraduate Honors Thesis Submitted In Partial Fulfillment Of The

Requirements For The Degree Of

Bachelor of Science

in

University Honors

and

Public Health: Health Science

Thesis Adviser

Dr. Claire Wheeler, MD PhD

Portland State University

2019 


\section{Abstract:}

Previous work has shown that elderly women with chronic or infectious disease are at an especially high risk for mental health disorders such as depression and anxiety ${ }^{1}$. Although, Nontuberculous mycobacterium (NTM) is a common lung disease in Oregon, little research has been published on fundamental epidemiological measures such as prevalence, or incidence, populations risks, and disease comorbidities ${ }^{234}$. The objective of the study is to build a rationale for screening for anxiety and depression in NTM patients through a review of current literature of other lung diseases. Additionally, this project includes a chart review that shows the proportion of patients with NTM who lack history of mental health diagnosis or medication prescription, but have high scores on the Patient Health Questionnaire (PHQ-8) and/or Generalized Anxiety Disorder (GAD-7), reflecting potentially significant symptomology of either depression, anxiety, or both. A cutoff score for "positive" vs. "negative" presence of each disorder was established, based on prior studies using these scales. Questionnaire data have been accumulated on patients in the study. These data were gathered, originally, for a larger biobank study by OHSU's Center for Infectious Disease team. In addition to the screening results, data regarding the patients' anxiety and/or depression diagnosis history and psychotherapeutic medication prescription history has been included in analysis. This has been done through a chart review of the patients' electronic medical records using the Epic system. Statistical analysis using a chi-square has helped ascertain if the prevalence of patients who show symptoms, but no intervention is significantly higher than in those who do not. For both depression and anxiety there is a statistically significant relationship ( $\mathrm{p}<0.05$ and $\mathrm{p}<0.001$ respectively) between 
screening score and the presence of a diagnosis/medication. Most participants who screened positive had both a diagnosis and took medications or they had neither. With these data I provide a recommendation that once patients are found positive for NTM, providers should screen for both depression and anxiety regardless of mental health history.

\section{Acknowledgements:}

The process of gathering and analyzing data has been a rewarding experience for me. I want to thank Dr. Emily Henkle. Without Dr. Henkle's faith in me a year ago, I wouldn't be writing about anxiety and depression in NTM patients. I also would have given up on research long ago, but the guiding hand she has given me has revitalized my passion for research. Overall, I want to thank the Infectious Disease team for allowing me to use their research for this project, but also for allowing me to partake in the research process. I want to especially thank them for allowing me to consent and deliver surveys to the patients of this study. Listening to the experiences of the patients would always make my day. Additionally, I would like to thank the woman who helped me the most in writing this thesis and gave me the confidence to keep writing, Dr. Claire Wheeler. The next person I want to thank is Professor Shifer for her help with analyzing my data. Finally, I want to thank my parents and grandparents. Every day I live and breathe I strive to make them proud and every day I am proud to be their daughter and granddaughter. To everyone who has helped me on this journey, I will be eternally grateful.

\section{Background/Literature Review}


What is Nontuberculous mycobacterium:

Nontuberculous mycobacterium (NTM) is a non-contagious, chronic, pulmonary lung condition. NTM is most commonly caused by the pathogenic complex Mycobacterium aviumintracellulare. However, dozens of bacteria that result in NTM have been documented. The mechanism of disease onset is currently unknown, although research has linked infection to vaporized water and airborne soil ${ }^{5}$.

Similar to tuberculosis (TB), chronic obstructive pulmonary disease (COPD), and cystic fibrosis (CF), the symptoms of NTM include shortness of breath (SOB), excessive coughing, fever and/or fatigue ${ }^{5}$. Although this disease affects mostly older people, for years patients are often misdiagnosed with other lung conditions such as asthma. In order to correctly diagnose this disease, two positive sputum samples or one bronchoscopy and a chest CT is needed. The disease displays itself differently among patients, which is part of the reason why it is often misdiagnosed. Some patients are asymptomatic and others, due to severe comorbidities and other clinical issues, may require oxygen. Another interesting differentiation between patients is that some patients may clear or contain the infection without further disease progression. For other patients, the disease will progress typically for several years causing chronic airway infection, destruction of lung tissue, shortness of breath, fatigue, decreased quality of life, and in some cases death. Treatment of this disease requires an antibiotic regime of 3-5 drugs for typically 1824 months ${ }^{5}$. Multiple drugs are required to control the symptoms and prevent disease spread, but the treatments are rarely curative. Additionally, the antibiotics often have irreversible negative side effects like the risk of hearing and vision loss. Finally, another major barrier to treating 
NTM is the high rate of comorbidities with other diseases such as COPD, bronchiectasis, and CF 5.

Due to minimal availability of epidemiological data and literature on NTM, few sources have obtained reports of the average age of NTM diagnosis, which is estimated to be around 69 years $^{26}$. Women have been found to be affected disproportionately more often than men; they comprise approximately $56 \%$ of patients with NTM $^{2}$. The prevalence of NTM in 2006 was twenty per one-hundred thousand ${ }^{7}$. Although, the prevalence was low, the incidence has been steadily increasing over the last decade ${ }^{2}$.

Anxiety and Depression in the General Population:

The CDC defines depressive disorder as a prolonged feeling of sadness that interferes with normal functioning of life ${ }^{8}$. The NIH defines anxiety disorder as prolonged feeling of uneasiness or worry that, like depression disorder, interferes with normal functioning of life ${ }^{9}$. According to the Anxiety and Depression Association of America, the prevalence of Major Depressive Disorder and Generalized Anxiety Disorder are 6.7\% and 3.1\% respectively ${ }^{10}$. Healthy geriatric patients have been shown to have a prevalence of depression $1-4 \%$. Women in the elderly population are found to have depression twice as often compared to elderly men ${ }^{11}$. One study also found that healthy geriatric patients have also shown a prevalence anxiety of $4.6 \%$, with only $36 \%$ being treated for it ${ }^{11}$. Anxiety, in elderly populations, have a prevalence of comorbidity with depression to be $38.6 \%$, which means it is not uncommon for patients to experience both ${ }^{12}$. Overall, with regards to risk, elderly patients who are single, younger, female, and low income are more likely to experience anxiety and depression symptoms ${ }^{12}{ }^{13}$. One of the 
reasons why mental health is studied in this population is that affective disorders like depression and anxiety have been shown to be major drivers of patient's health and quality of life ${ }^{14}$. Treatment for depression, as well as anxiety, is mainly pharmacotherapy and psychotherapy. Other forms of therapy like exercise, yoga, and music can also reduce anxiety and depression symptoms 1516 .

What started the conversation of mental health in NTM patients:

In 2015, the Center of Infectious Disease team (CIDS) at OHSU gathered together clinicians, patients, and providers from all around the state. They spent an entire day discussing the lack of research in NTM research and clinical care. One of the topics that was brought up was the need for assistance in lessening the impact of mental health for patients with NTM ${ }^{17}$. This had kickstarted the conversation surrounding mental health for this patient population and exemplified the need for research. Currently, there appears to be little or no research being done to determine the prevalence and impact of mental health disturbances in patients with NTM. This is the case, although depression and/or anxiety in bronchiectasis, TB, COPD, and CF are widely studied. To show the importance of studying mental health in this population, other lung disease research will be used as a baseline of information. The three lung diseases most like NTM are chronic obstructive pulmonary disease (COPD), cystic fibrosis (CF), and tuberculosis (TB). COPD is a chronic lung condition that causes obstructed airflow. CF is a chronic genetic disorder that allows thick mucus to get clogged in the patient's airways. TB, on the other hand, is the most similar lung condition to NTM, because it is a chronic infectious disease. TB and NTM are also similar with regards to patient clinical symptoms and signs, and chest radiograph results ${ }^{18}$. 


\section{Depression and Anxiety in Lung Disease Populations:}

Chronic disease, as well as lung disease populations have high rates of anxiety and depression. Depression in TB patients have been shown to be present in up to $70 \%$ of ambulatory populations and $80 \%$ in hospitalized populations ${ }^{14}$. It's also been found that depression and anxiety in TB patients has a prevalence of $43 \%$ and $41 \%$ respectively, with a comorbidity of both to be $40 \%{ }^{19}$. One study found that one in four COPD patients experience significant depression symptoms ${ }^{20}$. COPD patients are ten times more likely to experience anxiety symptoms or panic attacks compared to the general population ${ }^{20}$. Anxiety has shown to have a prevalence of about $40 \%$ in COPD patients ${ }^{20}$. For patients with interstitial lung disease, the prevalence of depression is $21 \%^{21}$. Anxiety, on the other hand has been observed in $31 \%$ of patients with interstitial lung disease ${ }^{22}$. Looking at CF patients, in one study $19 \%$ have shown depressive symptoms and $32 \%$ have shown anxiety symptoms. Overall, the symptom elevations were 2-3 times those of community samples ${ }^{23}$. With increasing symptom severity, the number of symptoms, and the amount of medications, comes an increasing rate of depression and anxiety 2021 . It is unclear what happens first, the lung disease symptoms severity or the depression, but with interstitial lung it is argued that it is actually health related quality of life, dyspnea, and lack of physical activity that leads to the mental disorder symptoms ${ }^{20}$.

Knowing that lung disease populations have high rates of anxiety and depression, there are several reasons why this is detrimental to the lives of these patients. Anxiety and depression have been shown to have a significant association with disease symptoms like pain and dyspnea severity ${ }^{21}$. When controlling for physical ailments, the presence of anxiety and depression were 
found to increase the cost of care by $45 \%$ in COPD patients ${ }^{20}$. COPD patients with these diagnoses are also more likely to have increased utilization rates and costs associated with their care during their visit at the hospital ${ }^{20}$. Along with an increased cost of care, the presence of depression in a COPD population is associated with an $12 \%$ increase in COPD-related physician visits, $2 \%$ higher number of annual hospitalizations, and $48 \%$ more COPD-related ER visits ${ }^{24}$. This same population is also more likely to have an increased length of stay at hospitals. Mental health has also been shown to affect patients' ability to self-manage disease ${ }^{25}{ }^{26}$. Although all of these are important factors that pulmonologists need to keep in mind when treating their lung disease patients, none of this matters if the patient isn't being adequately treated for their mental health. One study found that in COPD patients with depression, only 27-33\% were being treated for it ${ }^{25}$. Anxiety also has been shown to affect a large portion of patients not being treated for it 27 .

Overall, although no studies have determined it to be so, based on the risks for depression and anxiety for patients with similar lung diseases, NTM patients may have high risk of anxiety and depression. NTM patients are mostly elderly, female, with many medications and symptoms, all of which are risk factors for depression and anxiety. The importance of researching depression and anxiety in this patient population goes beyond helping patients feel better. Research suggests that depression and anxiety may impact treatment adherence, as suspected with coronary artery disease patients ${ }^{28}$. This is especially important since both CF and bronchiectasis patients have shown low treatment adherence over the course of several months ${ }^{29}$. CF patients tend to report high treatment burden due to the intensive measures required to take the medication, much like how involved NTM medication regimens are ${ }^{26}$. 
To help determine if patients experience affective disorder symptoms, providers and researchers use screening tools. The most common screening tools for anxiety are the Anxiety Inventory for Respiratory Disease (AIR) and the Generalized Anxiety Disorder Scale (GAD7). The most common screening tools for depression are the Hospital Anxiety and Depression Scale (HADS) and Patient Health Questionnaire Scale (PHQ-9). Although there are two scales that were specifically made for the elderly population; Geriatric Depression Scale (GDS-15) and Geriatric Anxiety Scale (GAS-10), since some NTM patients are not elderly, using the geriatric scales are not justified ${ }^{30}{ }^{31}$. This study will use the PHQ-8, a depression screening tool, and the GAD-7, an anxiety screening tool. Both screening tools, similar to other tools of the same purpose, have been shown to have high validity and reliability in screening for these two mental health diseases ${ }^{32}{ }^{33}$. The PHQ-8 gives a maximum value of 24 and the GAD-7 gives a maximum value of 21. A score greater than or equal to 10 is deemed diagnostic as seen from literature for the PHQ-8 and GAD-7 34 .

This thesis will determine if there is inadequate surveillance of mental health care from Primary care physicians (PCP's). Additionally, as explained in the abstract, this thesis will determine if there is a need for Pulmonologists to screen for depression/anxiety in NTM patients. The authors of one article, deemed the largest anxiety and depression screening to date for $\mathrm{CF}$ patients, recommends yearly screenings for both disorders ${ }^{35}$. Additionally, the US Preventative Task Force recommends that anyone over the age of 60 years old should have regular screening for depression. But this screening should be paired with an environment that can treat, diagnose, and follow up with the patient ${ }^{36}{ }^{37}$. The process of deciding who will screen the patients may be problematic since PCPs bear the greatest burden of diagnosing and treating mental health disorders in their patients, but the nature of primary care practice (high patient loads, short office 
visits, multiple diagnoses, etc.) may sometimes cause problems to fall through the cracks. Since mental health has been shown to affect disease outcomes like treatment adherence and success, all providers must make it a priority to discover what obstacles the patient may face that will prevent clinical success.

\section{Methods/Project Design:}

Main study methods:

The study took place at the Oregon Health and Science University (OHSU) Hospital's Pulmonology Medicine clinic. The two attending physicians were Dr. Kevin Winthrop and Dr. Luke Strnad. The consent and survey process took place at the clinic. Following consent, the research coordinator (myself and other IDS staff) gave the patients two surveys; The PHQ-8, and the GAD-7 scale. The original study was made to create a repository of NTM-positive patient information for multiple studies. The biobank repository allows the Infectious Disease staff to analyze and observe disease activity, quality of life, NTM module validity, and human specimen immune response for our NTM population. Although this thesis is primarily a chart review of cohort NTM patients, patient recruitment and screening will continue indefinitely, by both myself and OHSU IDS staff.

Thesis project: 
The data taken at enrollment was date of birth, ethnicity, gender, marital status, highest education level, GAD-7 score, and PHQ-8 score. The screening scores were stored in OHSU's HIPAA approved research database, REDcap. Patient information in REDcap was paired with a study ID. In order to connect the study ID with the patient's name and MRN, a separate password protected database was accessed.

During this chart review I looked into the patient's electronic medical records using Epic. Two things were searched for in Epic about depression and anxiety; history of diagnosis and psychotherapeutic medications. Since most of the patients do not maintain their primary care at OHSU, their medical records were shared to the OHSU Epic system, Care Everywhere. Diagnosis information was found in the "problems list", "resolved problems list", or "medical history" list. Anything posted in these categories on Epic require a diagnosis, prior to display. The presence of psychotherapeutic medications was found by going into the "Chart Review" section of Epic and finding the day of enrollment. By opening the provider's "Encounter summary", I was able to cross reference the list of medications they were taking with a list of the most common depression and anxiety medications. This list included both generic and brand name medications as shown from Table 1 and Table 2. The sources for this list include the FDA, the Mayo clinic, WebMD, Healthyplace, and Healthline, with every medication on the list being sourced from at least two places. For those with a diagnosis and a medication that was dualpurpose for anxiety or depression, I paired the medication intent with the diagnosis. For those with a dual-purpose medication, but no diagnosis, both anxiety and depression were labeled as the intent for the medication. 
Table 1. List of Depression Medications Used in Analysis

\begin{tabular}{|c|c|c|}
\hline Class type & Brand Name & Generic Name \\
\hline \multirow{6}{*}{$\begin{array}{l}\text { Selective Serotonin Reuptake } \\
\text { Inhibitors (SSRIs) }\end{array}$} & Celexa * & Citalopram * \\
\hline & Lexapro * & Escitalopram * \\
\hline & Paxil, Pexeva * & Paroxetine * \\
\hline & Prozac & Fluoxetine * \\
\hline & Luvox* & Fluvoxamine* \\
\hline & Zoloft * & Sertraline * \\
\hline \multirow{4}{*}{$\begin{array}{l}\text { Monoamine Oxidase Inhibitors } \\
\text { (MAOIs) }\end{array}$} & Emsam * & Selegiline * \\
\hline & Marplan * & Isocarboxzaid * \\
\hline & Nardil * & Parnate * \\
\hline & Phenelzine * & Tranylcypromine * \\
\hline \multirow{9}{*}{ Tricyclic Antidepressants } & Elavil, Endep, Levate & Amitriptyline \\
\hline & Asendin & Amoxapine \\
\hline & Norpramin, Pertofrane * & Desipramine * \\
\hline & Adapin, Silenor, Sinequan * & Doxepin * \\
\hline & Tofranil * & Imipramine * \\
\hline & Anafranil * & Clomipramine * \\
\hline & Aventyl, Pamelor & Nortriptyline \\
\hline & Vivactil & Protriptyline \\
\hline & Surmontil, Trimip, Tripramine & Trimipramine \\
\hline \multirow{7}{*}{ Atypical Antidepressants } & Ludimol & Maprotiline \\
\hline & Desyrel* & Trazodone * \\
\hline & & Nefazodone \\
\hline & Remeron & Mirtazapine \\
\hline & Viibryd & Vilazodone \\
\hline & Trintellix, Brintellix & Vortioxetine \\
\hline & $\begin{array}{l}\text { Wellbutrin, Wellbutrin, } \\
\text { Aplenzin, Forfivo XL, Budeprion } \\
\text { SR, Budeprion XL, Buproban, } \\
\text { Wellbutrin SR, Wellbutrin XR, } \\
\text { Zyban }\end{array}$ & Bupropion \\
\hline \multirow{4}{*}{$\begin{array}{l}\text { Selective Serotonin and } \\
\text { Norepinephrine Reuptake } \\
\text { Inhibitors (SNRI) }\end{array}$} & Cymbalta * & Duloxetine * \\
\hline & Effexor, Effexor XR * & Venlafaxine * \\
\hline & Pristiq, Khedezla & Desvenlafaxine \\
\hline & Fetzima & Levomilnacipran \\
\hline
\end{tabular}


Table 2. List of Anxiety Medications Used in Analysis

\begin{tabular}{|c|c|c|}
\hline Class type & Brand Name & Generic Name \\
\hline \multirow{6}{*}{ Benzodiazepines } & Xanax & Alprazolam \\
\hline & Librium & Chlordiazepoxide \\
\hline & Klonopin & Clonazepam \\
\hline & Valium & Diazepam \\
\hline & Ativan & Lorazepam \\
\hline & Serax & Oxazepam \\
\hline \multirow{3}{*}{ Anticonvulsants } & Depakote, Depakote ER & Divalproex \\
\hline & Neurontin & Gabapentin \\
\hline & Lyrica & Pregabalin \\
\hline \multirow{3}{*}{ Beta-Blockers } & Tenormin & Atenolol \\
\hline & Corgard & Nadolol \\
\hline & $\begin{array}{l}\text { Inderal, Betachron E-R, } \\
\text { InnoPran XL }\end{array}$ & Propranolol \\
\hline \multirow{4}{*}{ Antipsychotic } & Moban & Molindone \\
\hline & Zyprexa & Olanzapine \\
\hline & Seroquel & Quetiapine \\
\hline & Risperdal & Risperidone \\
\hline
\end{tabular}

Once medication use and diagnosis data were extracted, the access dataset was converted to a delimited comma-separated file and imported to Stata. Once in Stata the categorical marital status was recorded to become a dichotomous variable. Ethnicity, a once categorical variable, was also recorded to become dichotomous variable. For statistical analysis, a chi-square was taken for both anxiety and depression, between positive screening scores and the presence of a diagnosis or medication.

\section{$\underline{\text { Results: }}$}


As shown in Table 3, the participants are mostly white (89\%), with a partner (67\%), and have a variety of education levels. Although about fifty percent of the patient population don't have a college degree, the proportions between education levels listed are nearly the same proportion between categories. The percentage of those with a high school diploma, college degree, some college, and professional degrees are 29\%, 25\%, 26\%, and 20\% respectively. The average age is 70 years old, which classifies the patient population as "old or elderly" ${ }^{38}$. Another important factor is that the participants were disproportionately female, with $78 \%$ being female and $22 \%$ being male. The ethnicity, average age, and gender proportions in this study were consistent with previous research done in Oregon, as listed in the Literature review section. Finally, the average score for the GAD-7 and the PHQ- 8 are 4.5 and 5 respectively.

Table 3. Demographics

\begin{tabular}{|c|c|c|c|}
\hline & $\mathrm{N}(\%)$ & Mean (SD) & Range \\
\hline Anxiety Screening Score & & $4.00(4.00)$ & $(0-21)$ \\
\hline Depression Screening Score & & $4.50(5.09)$ & $(0-23)$ \\
\hline \multicolumn{4}{|l|}{ Highest Education Level } \\
\hline High school or less & $61(28.65)$ & & $(0,1)$ \\
\hline Some college & $55(25.82)$ & & $(0,1)$ \\
\hline Bachelor's degree & $70(25.35)$ & & $(0,1)$ \\
\hline Professional or graduate degree & $43(20.19)$ & & $(0,1)$ \\
\hline \multicolumn{4}{|l|}{ Race } \\
\hline White & $189(88.73)$ & & $(0,1)$ \\
\hline \multicolumn{4}{|l|}{ Marital Status } \\
\hline In a relationship & $142(66.66)$ & & $(0,1)$ \\
\hline \multicolumn{4}{|l|}{ Gender } \\
\hline Female & $166(77.93)$ & & $(0,1)$ \\
\hline Age & & $70.02(12.34)$ & $(27-95)$ \\
\hline Participants (n) & 213 & & \\
\hline
\end{tabular}


As demonstrated in Table 4, the proportion of patients who screened positive on the depression screening tool or anxiety screening tool were $20 \%$ and $14 \%$ respectively. Out of all the patients, $32 \%$ had a depression diagnosis and $28 \%$ were taking medication for depression. On the other hand, $34 \%$ had a diagnosis and $34 \%$ were taking medication for anxiety.

Table 4. Descriptive statistics of Anxiety and Depression

\begin{tabular}{lc}
\hline & $\mathrm{N}(\%)$ \\
\hline Screened Positive: & $30(14.00)$ \\
Anxiety & $42(19.70)$ \\
Depression & \\
Had diagnosis: & $72(33.80)$ \\
Anxiety & $68(31.92)$ \\
Depression & \\
Took medication: & $72(33.80)$ \\
Took Anxiety Medication at enrollment & $60(28.17)$
\end{tabular}

Table 5 demonstrates the difference in diagnosis and medication status between those who screened positive and those who screened negative for anxiety and depression. Within those who screened positive using the GAD-7, 43\% had both a diagnosis and were taking medication, $17 \%$ had just a diagnosis. $20 \%$ had neither, and $20 \%$ were just taking medications. On the other hand, of those who screened positive for the PHQ-8; 38\% had both a diagnosis and were taking medications, $14 \%$ had just a diagnosis, $2 \%$ were just taking medications, and $45 \%$ had neither. 
Table 5: Comparing Those Who Screened Positive For Depression v.s. Anxiety

\begin{tabular}{lrc}
\hline & $\mathrm{N}(\%)$ & $\begin{array}{c}\text { Mean } \\
\text { Score }\end{array}$ \\
\hline PHQ-8 >=10 & & \\
\hline Diagnosis and Medications & $16(38.10)$ & 12.81 \\
Just Diagnosis & $6(14.29)$ & 15.17 \\
Just Medications & $1(2.38)$ & 10.00 \\
Neither & $19(45.24)$ & 14.00 \\
\hline GAD-7 >=10 & & \\
\hline Diagnosis and Medications & $13(43.33)$ & 13.46 \\
Just Diagnosis & $5(16.67)$ & 13.20 \\
Just Medications & $6(20.00)$ & 15.67 \\
Neither & $6(20.00)$ & 15.17 \\
\hline
\end{tabular}

Table 6 shows the Chi-square of screening results and prescription/diagnosis status. The mean GAD-7 score for those who had a diagnosis and were prescribed medication were 5.71 and 6.06 respectively. On the other hand, the mean PHQ-8 score for those who had a diagnosis and were prescribed medication were 7.05 and 6.3 respectively. The number of those who screened positive on the PHQ-8 and had a diagnosis or had a depression medication prescription was 68 and 60 respectively. On the other hand, the number of those who screened positive on the GAD7 and had a diagnosis or had a depression medication prescription was 72 and 72 respectively. The chi-square showed that there is a significant relationship between PHQ-8 results and the presence of a diagnosis $(\mathrm{p}<0.05)$. Additionally, there was a significant relationship between the PHQ-8 results and the presence of a prescribed depression medication $(\mathrm{p}<0.001)$. The chi-square also showed that there is a significant relationship between GAD-7 results and the presence of a diagnosis $(\mathrm{p}<0.001)$. Finally, there is a significant relationship GAD-7 results and the presence of prescribed medication $(\mathrm{p}<0.001)$. 
Table 6: Chi-square of Sreening Results versus Diagnosed \& Perscribed Medication

\begin{tabular}{lcccc}
\hline & N & $\begin{array}{c}\text { N (\%) } \\
\text { Screened } \\
\text { Positive }\end{array}$ & Mean (SD) & $\begin{array}{l}\text { Chi-Square } \\
\text { P-value }\end{array}$ \\
\cline { 2 - 5 } & & & & \\
PHQ-8 & 60 & $17(40.00)$ & $6.30(4.93)$ & 0.000 \\
$\quad$ Perscribed Medication & 68 & $22(52.38)$ & $7.05(5.53)$ & 0.048 \\
$\quad$ Diagnosed & 72 & $19(63.33)$ & $6.06(5.52)$ & 0.000 \\
GAD-7 & 72 & $18(60.00)$ & $5.71(5.17)$ & 0.000 \\
$\quad$ Perscribed Medication & & & & \\
$\quad$ Diagnosed & & &
\end{tabular}

\section{Discussion:}

As seen from Table 4, a higher percentage of patients had a diagnosis or took medications for anxiety, compared to depression. But a greater proportion screened positive for depression, as opposed to anxiety. This suggests that patients were more likely to be depressed at enrollment. The screening tool is useful for determining whether or not someone has depressive symptoms, but is not absolutely diagnostic. There are several reasons why a greater portion have a diagnosis and medications for anxiety. Firstly, feelings of anxiousness are a common overarching symptom in chronic disease patients. Additionally, anxiety symptoms are objectively easier to identify compared to depressive symptoms. Also, symptoms for depression are often more subtle. Finally, because of the immediate connection to depression disorder and the stigma that inherently goes with saying someone is "sad" or feeling depressed", patients may feel less inclined to voluntarily offer up information surrounding this topic. Whereas with anxiety, expressing feelings of anxiousness or stress are more normalized, therefore a patient might offer information relating to this more freely. 
Additionally, a number of patients were given medications without a diagnosis for both anxiety and depression. There are three possible reasons for this. Firstly, there could be fewer depression medications that have dual purposes with other disorders compared to anxiety medications. Secondly, anxiety symptoms are more generalizable to many other disorders, such as Post-traumatic stress disorder, panic disorder, phobias, social anxiety disorder, and insomnia. Someone might take the medication for a different anxiety disorder, other than general anxiety disorder. Third, it's possible that the after initial evaluation, the symptoms presented differently for a different diagnosis than before ${ }^{39}$. Someone could be diagnosed for anxiety, then years later develop depression, but since the medication is often dual-purpose, they could use it for both. Fourth, maybe providers are prescribing psychotherapeutic medications without a diagnosis. Finally, patients may request that depression or anxiety not be listed as a diagnosis in their medical file, for fear of stigma.

To break down Table 5 further, when looking into those who scored positive in the GAD$7,80 \%$ had either a diagnosis or took medications, while $20 \%$ had neither. For those who screened positive on the PHQ-8, 56\% had either a diagnosis or took medications, while $45 \%$ had neither. A greater proportion of patients who scored positive for anxiety compared to depression, already had a diagnosis or prescription compared to those within the same group who didn't have an either of those things. Whereas, a greater proportion of those who scored positive for depression, compared to anxiety, have unrecognized depression with no intervention.

For those with seemingly unrecognized symptomology, there are several possible reasons for this. First, their PCP might not be regularly screening them. Second, the patient could have requested the information be removed from their medical record. Third, there might be records missing from the Care Everywhere and EPIC system from other institutions. 
Besides those with no clinical history, the other group who screened positive are those with medications or a diagnosis. It's important to note that EPIC does not provide information on other forms of therapy like talk therapy, Yoga, exercise, art therapy, or music therapy. It's possible that those with just a diagnosis declined taking medication, were using alternative therapy styles, or did nothing at all. For those who were taking medications without a diagnosis, since the medical records available during chart review were incomplete, the diagnosis may be missing in other medical record files. Additionally, the medication that the patient used at time of diagnosis needed modifications or supplemental therapies to do alongside the pharmaceutical. Finally, it's possible the patient wasn't taking the medication as intended so the desired effects were not presenting. Overall, those who screened positive and had a diagnosis or prescription psychotherapeutic need follow up for re-evaluation of treatment.

Table 6 demonstrated that the screening results for both anxiety and depression were dependent on the presence of a diagnosis or medication prescription. This shows that follow up with patients who have pre-existing symptoms is important for future mental health management.

\section{Limitations:}

There are several limitations to this study. First, when looking into the medications prescribed to patients, it was found that most of the time neither Care-everywhere, OHSU Epic system, or the Encounter summary from the provider would describe what the medication was used for. This is a limit because the data may overestimate those who took one type of 
psychotherapeutic medication over the other. While several charts showed indications attached to medications, it was a common trend for the indications to be absent. This also could have been done via the patients request for privacy. Ultimately it is better practice to always provide indications for medications to facilitate continuity of care and viability of these data for future research.

Second, the time of diagnosis was not listed in the patient's problem list, resolved problem list, or medical history list. Marking when the diagnosis or start of symptoms appeared for the patient not only can be helpful for providers, but also researchers who want to look into treatment success for mental health interventions. This is a limit to the study because the chart review process sometimes happened years after the enrollment date. Essentially, some patients could have been diagnosed after the screening date.

Third, it's possible that patients weren't taking their medications due to adverse side effects, complications with other medications, or polypharmacy from multiple illnesses that make keeping track difficult. Although there is no for sure way to determine if patients are taking their medications, this needs to be considered when discussing limitations.

Fourth, the Epic system does not list other forms of treatment that the patient might access, like talk therapy, yoga, meditation, and exercise. All of these are valid forms of treatment for anxiety or depression but can only be accessed through the use of survey, not chart review. This is a limitation because the study maybe underestimating the proportion of patients receiving treatment.

Finally, the usage of the PHQ-8 instead of PHQ-9 is potentially problematic. Most studies looking at depression use the PHQ-9 over the PHQ-8, therefore there is more research on the cutoff for a positive screening score in the PHQ-9. The PHQ-9, compared to PHQ-8, includes 
a question about suicidal ideations. The decision to use the PHQ-8 within the Infectious Disease lab was originally made because this lab doesn't have the ability to adequately follow up with patients if they indicate that they have suicidal ideations, as per state law.

Generalizability of results:

The demographic information gained from this study like average age and gender were similar to other studies in areas such as South Korea, Hawaii, the United States mainland, and Zambia. Overall, the average age of studied NTM populations seems to be between the ages of 60 and 70 years old. Additionally, in all of these locations women showed a higher prevalence, compared to men, for NTM diagnosis 404142 . This study and other Oregon NTM studies have a higher proportion of patients that are white, compared to other races. When looking at an article that specifically studied the epidemiology of the NTM in the United States, excluding Hawaii, Puerto Rico, and Alaska, the proportion of white participants was $70 \%$ compared to the $88 \%$ found from this study ${ }^{43}$. Oregon, compared to the rest of the United States, is very white which explains the result differences in ethnicity. Overall, these results are most generalizable to the Oregon NTM population and less so for the rest of the United States due to the lack of racial diversity.

\section{Possible Bias:}

The most apparent bias from this study is convenience selection bias and extraction researcher bias. This researcher extraction bias was mitigated by creating clear extraction 
procedures in regards where to find the data and by creating a set list of medications to cross reference. With regards to the selection bias, the patients needed to be recruited to OHSU from a different pulmonologist. There are several factors that could lead a patient to refuse the referral to OHSU such as difficulty reaching the location, cost, and availability.

Other possible biases that could have arose were procedural bias, measurement bias, and response bias. Procedural bias is possible because the questionnaire was filled out after the Pulmonology visit and right before the patient went in for a blood draw. Therefore, it is possible that they were rushing as they filled out the questionnaires. Measurement bias is possible because mental health is a personal topic that many Americans feel shame over. Since the questions are all about mental health, it's possible that patients underreported how often they felt those symptoms. Therefore, as said before, the current results could be underestimations of the true burden of mental health in the NTM populations. Although Response bias is always a possibility with surveys, this was mitigated by stating, during the consent process, the purpose of the experiment, rather than what we expect from the experiment.

\section{Conclusion:}

The prevalence, incidence, and impact of anxiety and depression in the NTM patient population has yet to be researched. Through this project, the prevalence of anxiety and depression, as well as the comorbidity of both was determined for one group of patients. With these data, I found that within this patient population, a higher proportion had a diagnosis or took a medication for anxiety, compared to depression. A higher proportion had a positive screening score for depression, compared to anxiety. The chi-square for both anxiety and depression showed a significant relationship between screening results and the presence of a diagnosis or 
prescription of medication. Overall, this patient population has mostly unrecognized anxiety and unresolved symptomatology in both disorders. Therefore, patients who screen positive need follow up for either a first-time diagnosis and treatment plan, or re-evaluation and additional support.

My recommendation from these results would be to screen all NTM patients every six months, which is the time period between most visits in the Infectious Disease clinic at OHSU. I also recommend collaborative care for patients suffering with mental illness, between the pulmonologist and primary care provider, and in some cases, with a mental health professional. Direct follow-up with either the patient or PCP is important once a patient screens positive for either of these diseases. Without follow up, the patient may continue living with unresolved symptoms. These very same symptoms are known to affect health outcomes, treatment adherence, and symptom severity in other lung disease populations, as described in the literature review.

In conclusion, more research on mental illness within this population needs to be done. As more cases of NTM are discovered, the Infectious Disease research team will continue to monitor these aliments in NTM patients, and hopefully provide appropriate referrals for effective treatments. For future projects the IDS team will look at screening scores over the life course of treatment plans, treatment adherence, and NTM symptomatology, in order to better understand mental health burden in this population. 


\section{References}

1. Lotfaliany M, Bowe SJ, Kowal P, Orellana L, Berk M, Mohebbi M. Depression and Chronic Diseases: Co-Occurrence and Communality of Risk Factors. J Affect Disord. 2018. doi:10.1016/j.jad.2018.08.011

2. Henkle E, Hedberg K, Schafer S, Novosad S, Winthrop KL. Population-based Incidence of Pulmonary Nontuberculous Mycobacterial Disease in Oregon 2007 to 2012. Ann Am Thorac Soc. 2015;12(5):642-647. doi:10.1513/AnnalsATS.201412-5590C

3. Cassidy PM, Hedberg K, Saulson A, McNelly E, Winthrop KL. Nontuberculous Mycobacterial Disease Prevalence and Risk Factors: A Changing Epidemiology. Clin Infect Dis. 2009;49(12):e124e129. doi:10.1086/648443

4. Novosad SA, Henkle E, Schafer S, et al. Mortality after respiratory isolation of nontuberculous mycobacteria a comparison of patients who did and did not meet disease criteria. Ann Am Thorac Soc. 2017;14(7):1112-1119. doi:10.1513/AnnalsATS.201610-8000C

5. Griffith DE, Aksamit T, Brown-Elliott BA, et al. An Official ATS/IDSA Statement: Diagnosis, Treatment, and Prevention of Nontuberculous Mycobacterial Diseases. Am J Respir Crit Care Med. 2007;175(4):367-416. doi:10.1164/rccm.200604-571ST

6. Adzic-Vukicevic T, Barac A, Blanka-Protic A, et al. Clinical features of infection caused by nontuberculous mycobacteria: 7 years' experience. Infection. 2018. doi:10.1007/s15010-018-1128-2

7. Winthrop KL, McNelley E, Kendall B, et al. Pulmonary nontuberculous mycobacterial disease prevalence and clinical features: An emerging public health disease. Am J Respir Crit Care Med. 2010;182(7):977-982. doi:10.1164/rccm.201003-05030C

8. American Psychiatric Association. Diagnostic and Statistical Manual of Mental Disorders. American Psychiatric Association; 2013. doi:10.1176/appi.books.9780890425596

9. NIMH » Anxiety Disorders. https://www.nimh.nih.gov/health/topics/anxietydisorders/index.shtml. Published 2018. Accessed May 30, 2019.

10. Facts \&amp; Statistics | Anxiety and Depression Association of America, ADAA. https://adaa.org/about-adaa/press-room/facts-statistics. Accessed May 24, 2019.

11. Alexopoulos GS. Depression in the elderly. In: Lancet. ; 2005. doi:10.1016/S0140-6736(05)666652

12. Van Der Veen DC, Van Zelst WH, Schoevers RA, Comijs HC, Oude Voshaar RC, Pachana NA. Comorbid anxiety disorders in late-life depression: Results of a cohort study. Int Psychogeriatrics. 2015. doi:10.1017/S1041610214002312 
13. Ahmed D, El Shair IH, Taher E, Zyada F. Prevalence and predictors of depression and anxiety among the elderly population living in geriatric homes in Cairo, Egypt. $J$ Egypt Public Health Assoc. 2014. doi:10.1097/01.EPX.0000455729.66131.49

14. Santos APC dos, Lazzari TK, Silva DR. Health-Related Quality of Life, Depression and Anxiety in Hospitalized Patients with Tuberculosis. Tuberc Respir Dis (Seoul). 2017;80(1):69. doi:10.4046/trd.2017.80.1.69

15. Duan-Porter W, Coeytaux RR, Mcduffie J, et al. Evidence Map of Yoga for Depression, Anxiety, and Posttraumatic Stress Disorder. doi:10.1123/jpah.2015-0027

16. Shirani Bidabadi S, Mehryar A. Music therapy as an adjunct to standard treatment for obsessive compulsive disorder and co-morbid anxiety and depression: A randomized clinical trial. $J$ Affect Disord. 2015;184:13-17. doi:10.1016/J.JAD.2015.04.011

17. Henkle E, Aksamit T, Barker A, et al. ANNALSATS SUPPLEMENT Patient-Centered Research Priorities for Pulmonary Nontuberculous Mycobacteria (NTM) Infection An NTM Research Consortium Workshop Report. 2016. doi:10.1513/AnnalsATS.201605-387WS

18. Kendall $B A$, Varley $C D$, Choi $D$, et al. Distinguishing tuberculosis from nontuberculous mycobacteria lung disease, Oregon, USA. Emerg Infect Dis. 2011;17(3):506-509. doi:10.3201/eid1703.101164

19. Duko B, Gebeyehu A, Ayano G. Prevalence and correlates of depression and anxiety among patients with tuberculosis at WolaitaSodo University Hospital and Sodo Health Center, WolaitaSodo, South Ethiopia, Cross sectional study. BMC Psychiatry. 2015;15(1):214. doi:10.1186/s12888-015-0598-3

20. Panagioti M, Scott C, Blakemore A, Coventry PA. Overview of the prevalence, impact, and management of depression and anxiety in chronic obstructive pulmonary disease. Int J Chron Obstruct Pulmon Dis. 2014;9(1):1289-1306. doi:10.2147/COPD.S72073

21. Ryerson CJ, Arean PA, Berkeley J, et al. Depression is a common and chronic comorbidity in patients with interstitial lung disease. Respirology. 2012. doi:10.1111/j.1440-1843.2011.02122.x

22. Holland AE, Fiore JF, Bell EC, et al. Dyspnoea and comorbidity contribute to anxiety and depression in interstitial lung disease. Respirology. 2014. doi:10.1111/resp.12360

23. Grossoehme DH, Szczesniak RD, Britton LL, et al. Adherence Determinants in Cystic Fibrosis: Cluster Analysis of Parental Psychosocial, Religious, and/or Spiritual Factors. Ann Am Thorac Soc. 2015;12(6):838-846. doi:10.1513/AnnalsATS.201408-3790C

24. Dalal AA, Shah M, Lunacsek O, Hanania NA. Clinical and Economic Burden of Depression/Anxiety in Chronic Obstructive Pulmonary Disease Patients within a Managed Care Population. COPD J Chronic Obstr Pulm Dis. 2011;8(4):293-299. doi:10.3109/15412555.2011.586659

25. Pooler A, Beech R. Examining the relationship between anxiety and depression and exacerbations of COPD which result in hospital admission: a systematic review. Int J Chron Obstruct Pulmon Dis. 2014;2014:315. doi:10.2147/COPD.S53255

26. Sawicki GS, Sellers DE, Robinson WM. High Treatment Burden in Adults with Cystic Fibrosis: Challenges to Disease Self-Management. doi:10.1016/j.jcf.2008.09.007 
27. Maurer J, Rebbapragada V, Borson S, et al. Anxiety and Depression in COPD * : Current Understanding, Unanswered Questions, and Research Needs. 2008. doi:10.1378/chest.08-0342

28. van Montfort E, Denollet J, Widdershoven J, Kupper N. Interrelation and independence of positive and negative psychological constructs in predicting general treatment adherence in coronary artery patients - Results from the THORESCI study. J Psychosom Res. 2016;88:1-7. doi:10.1016/j.jpsychores.2016.06.009

29. McCullough AR, Tunney MM, Quittner AL, Elborn JS, Bradley JM, Hughes CM. Treatment adherence and health outcomes in patients with bronchiectasis. BMC Pulm Med. 2014;14(1):107. doi:10.1186/1471-2466-14-107

30. Smarr KL, Keefer AL. Measures of depression and depressive symptoms: Beck Depression Inventory-II (BDI-II), Center for Epidemiologic Studies Depression Scale (CES-D), Geriatric Depression Scale (GDS), Hospital Anxiety and Depression Scale (HADS), and Patient Health Questionna. Arthritis Care Res. 2011;63(SUPPL. 11):454-466. doi:10.1002/acr.20556

31. Kneebone II, Fife-Schaw C, Lincoln NB, Harder H. A study of the validity and the reliability of the Geriatric Anxiety Inventory in screening for anxiety after stroke in older inpatients. Clin Rehabil. 2016;30(12):1220-1228. doi:10.1177/0269215515619661

32. Löwe B, Decker O, Müller S, et al. Validation and Standardization of the Generalized Anxiety Disorder Screener (GAD-7) in the General Population. Med Care. 2008;46(3):266-274. doi:10.1097/MLR.0b013e318160d093

33. Cameron IM, Crawford JR, Lawton K, Reid IC. Psychometric comparison of PHQ-9 and HADS for measuring depression severity in primary care. Br J Gen Pract. 2008;58(546):32-36. doi:10.3399/bjgp08X263794

34. Kroenke K, Strine TW, Spitzer RL, Williams JBW, Berry JT, Mokdad AH. The PHQ-8 as a measure of current depression in the general population. J Affect Disord. 2009;114(1-3):163-173. doi:10.1016/j.jad.2008.06.026

35. Quittner AL, Goldbeck L, Abbott J, et al. Prevalence of depression and anxiety in patients with cystic fibrosis and parent caregivers: Results of the International Depression Epidemiological Study across nine countries. Thorax. 2014;69(12):1090-1097. doi:10.1136/thoraxjnl-2014-205983

36. Pignone MP, Gaynes BN, Rushton JL, et al. Screening for Depression in Adults: A Summary of the Evidence for the U.S. Preventive Services Task Force. Ann Intern Med. 2002;136(10):765. doi:10.7326/0003-4819-136-10-200205210-00013

37. Vieira ER, Brown E, Raue P. Depression in older adults: Screening and referral. J Geriatr Phys Ther. 2014;37(1):24-30. doi:10.1519/JPT.0b013e31828df26f

38. WHO | Proposed working definition of an older person in Africa for the MDS Project. WHO. 2016. https://www.who.int/healthinfo/survey/ageingdefnolder/en/. Accessed May 29, 2019.

39. Current Diagnosis and Treatment of Anxiety Disorders. https://www.ncbi.nlm.nih.gov/pmc/articles/PMC3628173/pdf/ptj3801030.pdf. Accessed May 29, 2019.

40. Chanda-Kapata P, Kapata N, Klinkenberg E, et al. Non-tuberculous mycobacteria (NTM) in Zambia: prevalence, clinical, radiological and microbiological characteristics. 2011. 
doi:10.1186/s12879-015-1264-6

41. Kim H-J, Lee JH, Yoon SH, et al. Nontuberculous mycobacterial pulmonary disease diagnosed by two methods: a prospective cohort study. doi:10.1186/s12879-019-4078-0

42. Adjemian J, Frankland TB, Daida YG, et al. Epidemiology of Nontuberculous Mycobacterial Lung Disease and Tuberculosis, Hawaii, USA. Emerg Infect Dis. 2017;23(3):439-447. doi:10.3201/eid2303.161827

43. Spaulding $A B$, Lai $Y L$, Zelazny $A M$, et al. Geographic distribution of nontuberculous mycobacterial species identified among clinical isolates in the United States, 2009-2013. Ann Am Thorac Soc. 2017;14(11):1655-1661. doi:10.1513/AnnalsATS.201611-8600C 